\title{
Effect of gastro-oesophageal reflux on upper oesophageal sphincter motility in children
}

\author{
J Willing, G P Davidson, J Dent, I Cook
}

\begin{abstract}
Motor events of the upper oesophageal sphincter associated with gastro-oesophageal reflux were evaluated in $\mathbf{5 3}$ symptomatic children (median age 13 months) who were studied recumbent and unsedated. Children were divided into four groups according to symptoms, and then into two groups according to the presence or absence of neurological deficit. No grouping had basal upper oesophageal sphincter pressure that differed significantly from any other. Oesophageal distention due to gastro-oesophageal reflux, which was recognisable as oesophageal common cavity episodes, was associated with augmentation of mean basal upper oesophageal sphincter pressure from 36.5 (SD 18) $\mathrm{mm} \mathrm{Hg}$ to 48.5 (18) $\mathrm{mm}$ $\mathrm{Hg}(\mathrm{p}<0.0001)$, irrespective of whether gastrooesophageal reflux caused oesophageal acidification. Abrupt relaxations of the upper oesophageal sphincter independent of swallowing and lasting up to three seconds occurred during $54 \%$ of common cavity episodes. Forty nine per cent of these relaxations occurred within four seconds after the onset of distention. The oesophageal distention caused by gastro-oesophageal reflux is a potent stimulus of transient upper oesophageal sphincter relaxations in children. These relaxations are a more likely explanation for oesophagopharyngeal reflux than defective basal upper oesophageal sphincter tone.
\end{abstract}

(Gut 1993; 34: 904-910)

Oesophagopharyngeal reflux is a common problem, especially in infants and can have serious consequences. Major complications that can occur include calorie loss due to vomiting with resultant growth failure and respiratory disease secondary to aspiration of refluxate.

The upper oesophageal sphincter (UOS) is

Gastroenterology Unit, Adelaide Children's Hospital, 72 King William Road, Adelaide, South

Australia

JWilling

G P Davidson

Gastroenterology Unit, Royal Adelaide Hospital, North Terrace, Adelaide, South Australia

J Dent

I Cook

Correspondence to:

Dr G P Davidson,

Gastroenterology Unit,

Adelaide Children's Hospital

72 King William Road, North

Adelaide, South Australia

5006

Accepted for publication 20 October 1992 considered to be the major barrier preventing oesophagopharyngeal reflux. The concept that oesophagopharyngeal reflux occurs because of abnormally low basal UOS tone has dominated thinking about its pathogenesis. ${ }^{1}$ There is minimal information about patterns of UOS pressure in children with problems believed to be related to oesophagopharyngeal reflux. Neither Sondheimer ${ }^{2}$ nor Staiano et $a l^{3}$ found UOS pressure to be any different in children with gastro-oesophageal reflux compared with those without. They examined the hypothesis that basal UOS hypotonia caused oesophagopharyngeal reflux in an indirect manner. The methods used did not allow continuous monitoring of UOS pressure or examination of motor events associated with occurrence of either
TABLE I Major presenting symptoms

\begin{tabular}{|c|c|c|}
\hline \multirow[b]{2}{*}{ Symptom } & \multicolumn{2}{|l|}{ No of patients } \\
\hline & $\begin{array}{l}\text { Without } \\
\text { neurological } \\
\text { deficit }(n=42)\end{array}$ & $\begin{array}{l}\text { With } \\
\text { neurological } \\
\text { deficit }(n=11)\end{array}$ \\
\hline Vomiting & 27 & 7 \\
\hline Irritability & 23 & 4 \\
\hline Recurrent respiratory disease & 3 & 3 \\
\hline Failure to thrive & 2 & 2 \\
\hline Abdominal pain & 4 & 1 \\
\hline Apnoea & 5 & 0 \\
\hline Food refusal & 7 & 1 \\
\hline Swallowing difficulties & 1 & 1 \\
\hline
\end{tabular}

Some patients presented with more than one major symptom.

gastro-oesophageal reflux or oesophagopharyngeal reflux. The capacity to monitor UOS pressure for prolonged periods has only recently been developed. Initially, a sleeve sensor was adapted for this purpose in adults ${ }^{45}$ and we have now developed the UOS sleeve sensor so that it is small enough for use in children. ${ }^{6}$

In this study we aimed to record for the first time patterns of UOS motility associated with occurrence of gastro-oesophageal reflux in children referred for evaluation of suspected disorders of oesophageal motility including pathological gastro-oesophageal reflux. We sought to investigate the hypothesis that episodes of spontaneous gastro-oesophageal reflux cause reflex UOS relaxations, which may be the mechanism of regurgitation rather than a deficiency of basal UOS tone.

\section{Patients and methods}

PATIENTS

Fifty five children aged 2 to 81 months (median 13 months) were enrolled in the study. The patients were referred to the gastroenterology unit of the Adelaide Children's Hospital for evaluation of symptoms thought to be due to gastro-oesophageal reflux or a feeding disorder. All children were fully assessed clinically by one of us (GPD) or one other consultant pediatric gastroenterologist. The study protocol was approved by the ethics research committee of Adelaide Children's Hospital. Informed parental consent was obtained before the study. Table I gives the classification of major presenting symptoms and incidence of neurological dysfunction in the 53 children in whom technically satisfactory UOS recordings were obtained. Neurological dysfunction was defined by the presence of symptoms and signs of cerebral palsy or developmental delay confirmed by the Denver developmental screening test.? 


\section{PROTOCOL}

Dual sleeve manometric assemblies were used for concurrent monitoring of lower oesophageal sphincter (LOS) and UOS pressures. Assemblies were passed transnasally without the aid of sedation after a three hour fast. After positioning of a $\mathrm{pH}$ electrode and manometric assembly (see later) the child was allowed to settle and was then fed appropriately for age with formula or nonacid food such as sandwiches and milk. Monitoring of spontaneous patterns of motility and oesophageal $\mathrm{pH}$ was started at the end of the meal and continued for four hours with the children recumbent and unsedated.

\section{RECORDING TECHNIQUE}

We have described the technique previously. ${ }^{6}$ Briefly, one of two manometric assemblies was used (Fig 1). These had different intersleeve distances to cope with the range of intersphincteric distances found in this age group. They had an outer diameter of $3.5 \mathrm{~mm}$ except for the proximal sleeve, which had an oval cross section $(3.5 \mathrm{~mm} \times 5.5 \mathrm{~mm})$. This allowed consistent positioning in the UOS in the anterior or posterior orientation, thus controlling for radial asymmetry of the pressure profile. ${ }^{4} \mathrm{Six}$ sideholes, perfused at $0.15 \mathrm{ml} / \mathrm{min}$, monitored gastric and oesophageal body pressures; one sidehole perfused at $0.07 \mathrm{ml} / \mathrm{min}$ monitored pharyngeal pressures. The two sleeves were perfused at $0.3 \mathrm{ml} / \mathrm{min}$. Distal oesophageal $\mathrm{pH}$ was monitored concurrently with a miniature intraluminal monopolar glass electrode (Microelectrodes Inc, New Hampshire, USA, model MI-506), and a skin electrode used as a reference (Micro-electrodes Inc, New Hampshire, USA, model MI-402)

The manometric assembly was positioned such that the side holes at each end of the LOS sleeve showed gastric and oesophageal body pressure patterns whereas the side holes at either end of the UOS sleeve indicated pharyngeal and

\section{assemblies used for}

recording. Bars= sleeve sensors; closed circles $=$ sidehole sensors; open circles $=p H$ electrodes.

$$
\text { UOS }
$$

Sleeve

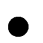

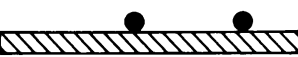

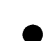
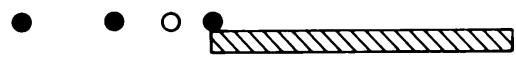
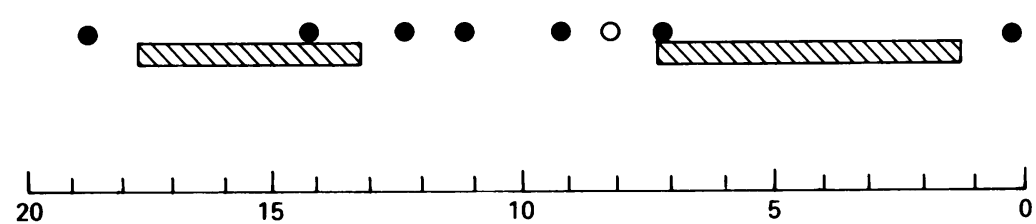

cm

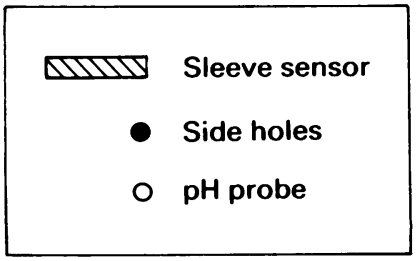

oesophageal body pressures (Figs 1, 2, 3). In two children the intersleeve distance was not compatible with simultaneous, technically satisfactory recording from the UOS and LOS. In these children the assembly was positioned to meet the clinical requirement for adequate LOS manometry. Consequently the data from the 53 children with technically satisfactory UOS recordings were analysed.

\section{DATA ANALYSIS}

Analysis of manometric tracings

Manometric indicators of reflux - Abrupt, sustained increases in intra-oesophageal pressure to intragastric pressure known as common cavity episodes, were used to recognise oesophageal distension by reflux without reference to the $\mathrm{pH}$ recording (Fig 2). Common cavity episodes have been described and evaluated in detail elsewhere ${ }^{8}$ and have been shown by fluoroscopy to be due to oesophageal body distention with gas. Common cavity episodes were only scored when the rise in intra-oesophageal pressure was recorded in at least two oesophageal body manometric channels. Rises in basal oesophageal pressure due to straining or breath holding were excluded by recognition of similar rises in the gastric pressure tracing, whereas true oesophageal body common cavity episodes were not associated with a comparable change of intragastric pressure (Fig 2).

Analysis of basal UOS pressure - For the purposes of this study basal UOS pressure was defined as the mean UOS pressure after editing of disturbances caused by strains or swallows. A strain was defined as an abrupt rise in gastric pressure of $5 \mathrm{~mm} \mathrm{Hg}$ or more above the baseline for two or more seconds, with a temporally associated disturbance of oesophageal body pressure. Swallowing was indicated by characteristic pharyngeal pressure waves in the most proximal sidehole (Figs 2,3). The reference point for timing of swallows was taken from the onset of this pressure wave. Basal UOS pressure was referenced to basal end expiratory midoesophageal body pressure and analysed in two ways as outlined:

(1) Basal UOS pressure was determined for every 12th minute throughout the monitoring period as described previously. ${ }^{6}$ Disturbances induced by swallowing were edited from the UOS pressure tracing by excluding pressures from four seconds before to six seconds after swallows. A visual mean of basal UOS pressure was determined from each edited minute of tracing if there were more than 14 seconds remaining after editing. In view of our recent finding that basal UOS pressure is influenced by the child's state of arousal, ${ }^{6}$ this was assessed throughout the monitoring period and noted as: A - resting with eyes shut, $B$ - resting with eyes open, C - moving, comfortable, D - moving, uncomfortable, and E - crying. The child's arousal classification was noted for each 12th minute value of UOS pressure and a mean value obtained for arousal states A and B. Although arousal states $C$ to $E$ were noted their data were not analysed for this study. 
Figure 2: Segment of manometric tracing showing pharynx, UOS, oesophageal body, LOS, and gastric pressures. The bottom tracing is intraoesophageal $p H$ recorded $3 \mathrm{~cm}$ above the lower oesophageal sphincter. The first pressure spike in the pharyngeal tracing indicates a normal swallow initiating a normal oesophageal body peristaltic wave simultaneous with LOS relaxation. After this sequence LOS pressure is re-established for about 10 seconds before there is a transient LOS relaxation that is associated with an oesophageal body common cavity episode and oesophageal acidification.
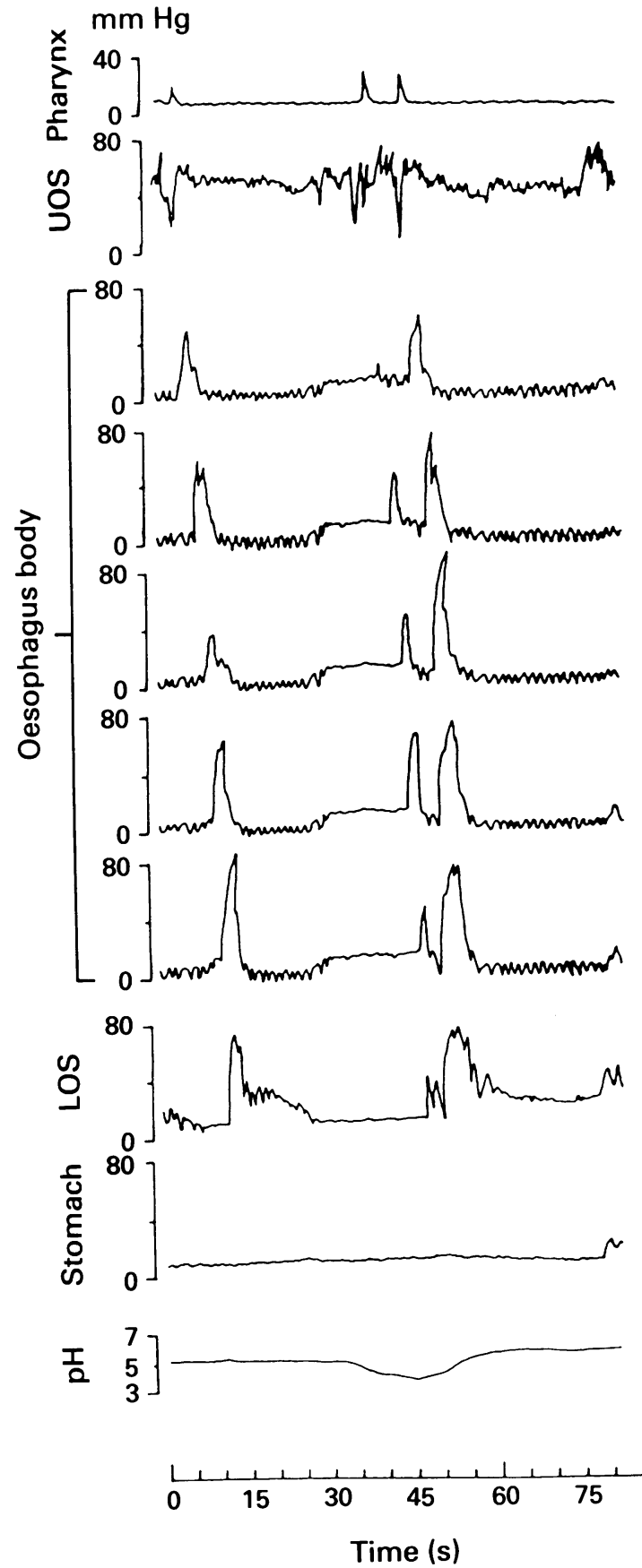

(2) UOS pressure around common cavity episodes was analysed if there were no swallows for 13 seconds before and three seconds after the onset of each common cavity episode (Fig 3). Visual mean values of UOS pressure were taken for each two second interval from 10 seconds before the onset of the common cavity episode until one second before the first swallow after the common cavity episode, or the onset of the first secondary oesophageal peristaltic wave (Fig 3). An overall value of basal UOS pressure was derived for the six seconds before and up to six seconds after the onset of the common cavity episode by averaging the individual two second values. Mean values for pre- and post-common cavity pressures were then derived for each child.

Measures of magnitude, duration, and extent of oesophageal distention - Occurrence of a pattern of intraoesophageal pressure oscillation identical to that of intragastric pressure during a common cavity episode was taken to indicate a period of communication between the lumina of the oesophageal body and stomach. The percentage of time that such a pattern was seen during common cavity episodes was recorded. The change of basal end expiratory intraoesophageal pressure produced by a common cavity episode was measured from an oesophageal body channel. The extent of an oesophageal common cavity was determined from the number of sideholes proximal to the LOS that registered the common cavity episode.

Transient UOS relaxations - The tracings were scanned for UOS relaxations that occurred independently of swallowing. This excluded any relaxation that had its onset within three seconds before or one second after the onset of a swallow. Figure 3 shows the analysis of transient UOS relaxations. These were scored when UOS pressure dropped to less than $50 \%$ of the prevailing UOS pressure within one second without the occurrence of swallowing or straining. In the case of relaxations that occurred before common cavity episodes, the reference basal UOS pressure was derived from the 10 seconds before the onset of the common cavity episode. For relaxations that occurred during common cavity episodes, the reference basal UOS pressure was derived from the UOS pressure values during the common cavity. Transient UOS relaxations were taken as having ended when UOS pressure rose above $50 \%$ of the relaxation pressure.

The nadir of transient UOS relaxations was the most clearcut point and so was related to the time of onset of the common cavity episode to the nearest second. Nadir pressure was referenced to the prevailing midoesophageal body pressure in that second and so took into account changes of basal oesophageal body pressure produced by common cavity episodes. The duration of the nadir of transient UOS relaxations was measured to the nearest second.

\section{Analysis of $p H$ recording}

Acid reflux was defined as a fall of oesophageal $\mathrm{pH}$ to four or less for four or more seconds. The $\mathrm{pH}$ changes associated with each common cavity episode were evaluated by taking the $\mathrm{pH}$ five seconds before and five seconds after the onset of the common cavity episode. The $\mathrm{pH}$ nadir during the common cavity episode was also noted.

\section{STATISTICAL ANALYSIS}

Student's paired $t$ test was used to compare basal UOS pressure for the six seconds before the common cavity to the six seconds after the onset of the common cavity. This was done by calculating mean deltas of UOS pressure for each subject and comparing group mean differences before and after the common cavity.

Incidence of transient UOS relaxations among symptom groups and between neurological divisions was compared by $\chi^{2}$ analysis. Incidence of transient UOS relaxations was compared with oesophageal acidification, change of oesophageal body pressure, and extent of communication by $\chi^{2}$ tests. 


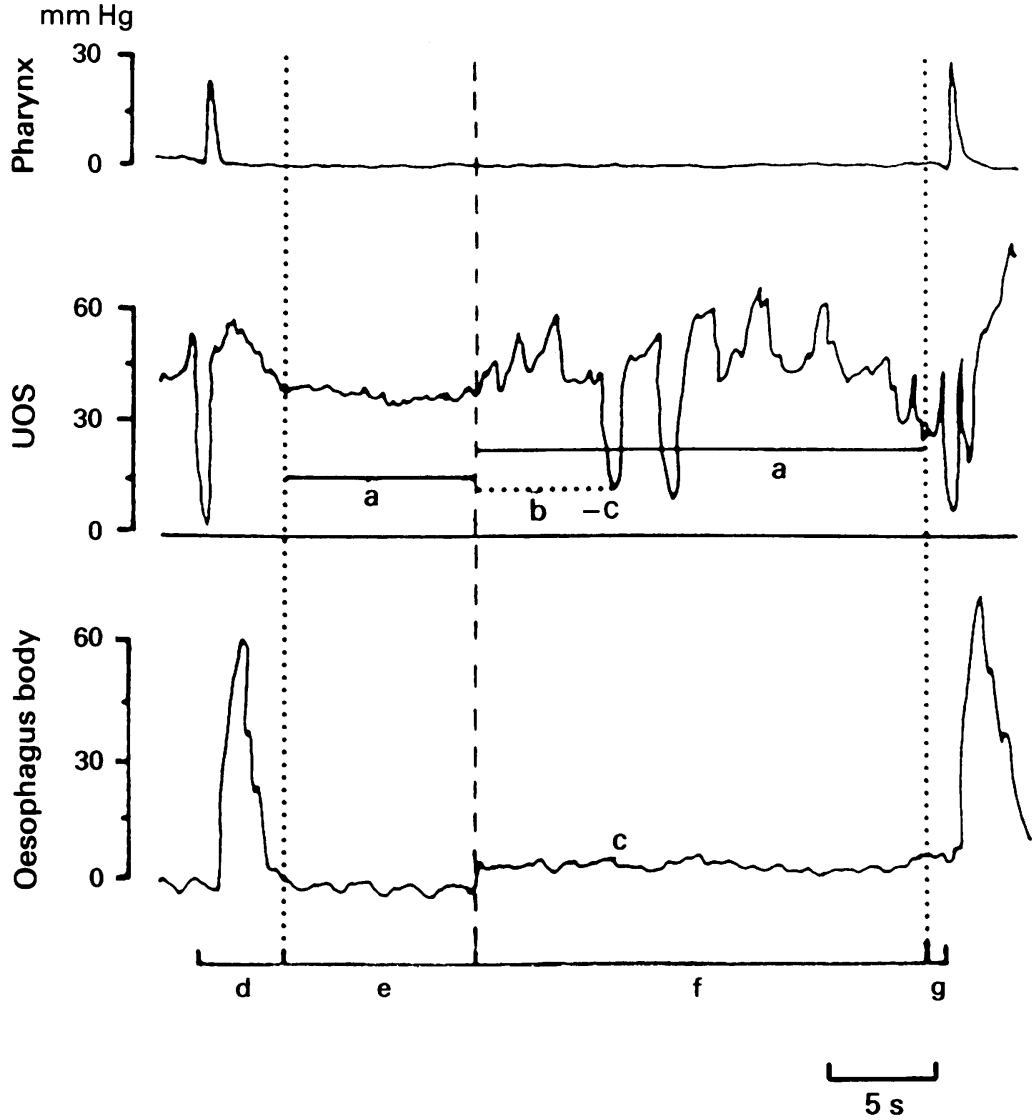

Figure 3: Measurement of transient $U O S$ relaxations. The spikes in the pharynx pressure tracing are generated by swallows. $a=$ $50 \%$ of UOS pressure referenced to basal end expiratory oesophageal body pressure before the common cavity onset; $b=$ timing of transient UOS relaxation, $c=$ prevailing oesophageal body pressure; $d=$ excluded time interval after a swallow; $e=10$ second time interval preceding the common cavity onset from which UOS pressures were measured; $f=$ interval after the common cavity onset from which UOS pressures were measured. This period ranged from 2 to 42 seconds; $g=e x c l u d e d$ time interval preceding a swallow.
The difference in increase in UOS pressure for those episodes with acid reflux and those without was compared with one way ANOVA.

A $p \times q$ factorial experiment with adjustment for unequal cell frequencies was used for comparison of basal UOS pressure among symptom groups, the presence or absence of neurological deficit, and the influence of arousal. An average of six values was available for estimation of a representative cell observation. ${ }^{10}$

TABLE II Relation between symptomatology and basal UOS pressure

\begin{tabular}{|c|c|c|}
\hline \multirow[b]{2}{*}{ Symptom group } & \multicolumn{2}{|c|}{ Basal UOS pressure (mm $\mathrm{Hg}$} \\
\hline & $\begin{array}{l}\text { Arousal } \\
\text { state } A \\
\text { Mean }(S D)\end{array}$ & $\begin{array}{l}\text { Arousal } \\
\text { state B } \\
\text { Mean }(S D)\end{array}$ \\
\hline Vomiting/failure to thrive & $\begin{array}{c}15 \cdot 4(11 \cdot 2) \\
(n=20)\end{array}$ & $\begin{array}{c}23 \cdot 3(21 \cdot 1)^{\star} \\
(n=14)\end{array}$ \\
\hline Irritability/abdominal pain & $\begin{array}{r}16 \cdot 2(7 \cdot 8) \\
(n=24)\end{array}$ & $\begin{array}{l}23 \cdot 3(16 \cdot 7)^{\star} \\
(\mathrm{n}=18)\end{array}$ \\
\hline $\begin{array}{l}\text { Recurrent respiratory disease/ } \\
\text { apnoea }\end{array}$ & $\begin{array}{c}20 \cdot 0(4 \cdot 3) \\
(n=3)\end{array}$ & $\begin{array}{l}26 \cdot 0(3 \cdot 3)^{\star} \\
(\mathrm{n}=3)\end{array}$ \\
\hline $\begin{array}{l}\text { Food refusal/swallowing } \\
\text { difficulties }\end{array}$ & $\begin{array}{c}12 \cdot 0(5 \cdot 6) \\
(n=4)\end{array}$ & $\begin{array}{c}29 \cdot 8(19 \cdot 0)^{\star} \\
(n=4)\end{array}$ \\
\hline
\end{tabular}

*Significant differences between arousal classifications. For this analysis, patients were grouped without reference to neurological state and according to the most prominent symptom. Values were not available for both arousal states in every child Values were not available for both arousal states in every
because of variation in behaviour pattern. No significant differences were found between symptom groups within arousal differences we
classifications.

TABLE III Relation between neurological deficit and basal UOS pressure

\begin{tabular}{lll}
\hline & \multicolumn{2}{l}{ Basal UOS pressure $(\mathrm{mm} \mathrm{Hg})$} \\
\cline { 2 - 3 } & $\begin{array}{l}\text { Arousal state A } \\
\text { Mean }(S D)\end{array}$ & $\begin{array}{l}\text { Arousal state B } \\
\text { Mean }(S D)\end{array}$ \\
\hline Without deficit & $16 \cdot 1(9 \cdot 2)(\mathrm{n}=40)$ & $24 \cdot 1(18 \cdot 7)^{\star}(\mathrm{n}=31)$ \\
Without deficit & $10 \cdot 7(9 \cdot 6)(\mathrm{n}=11)$ & $23 \cdot 4(17 \cdot 7)^{\star}(\mathrm{n}=8)$ \\
\hline
\end{tabular}

$\star$ Significant differences between arousal classifications. Values were not available for both arousal groups in every child because of variations in behaviour pattern.
Differences among nadir pressures of transient UOS relaxations occurring before the onset of the common cavity, and the first, second, and third and subsequent transient UOS relaxations after the onset of the common cavity were analysed as a single factor experiment with adjustment for unequal sample size. ${ }^{10}$

All data are expressed as median and interquartile ranges unless otherwise stated.

\section{Results}

BASAL UOS PRESSURE INDEPENDENT OF REFLUX Tables II and III show the mean values for basal UOS pressure according to arousal state, symptom classification, and presence or absence of neurological deficit. No significant differences were evident among the different clinical groups for arousal states A or B. Mean basal UOS pressure was not significantly different in the patients with neurological deficit compared with the neurologically normal children. Consistent with our previous report ${ }^{6}$ arousal state influenced UOS pressure irrespective of the clinical grouping, with UOS pressure being significantly greater in arousal state $B$ in all cases $(p<0.001)$.

BASAL UOS PRESSURE ASSOCIATED WITH COMMON CAVITY EPISODES

Screening of the tracings showed 369 common cavity episodes. The median number identified per child was five, with a wide variation from zero to 29. Of these, 102 episodes fitted the criteria described in the methods section, which were designed to ensure that the effects of swallowing or straining did not influence assessment of the effect of reflux on UOS pressures. These episodes gave a total of 551 two second time intervals before the onset of the common cavity episodes, and 476 two second time inter-

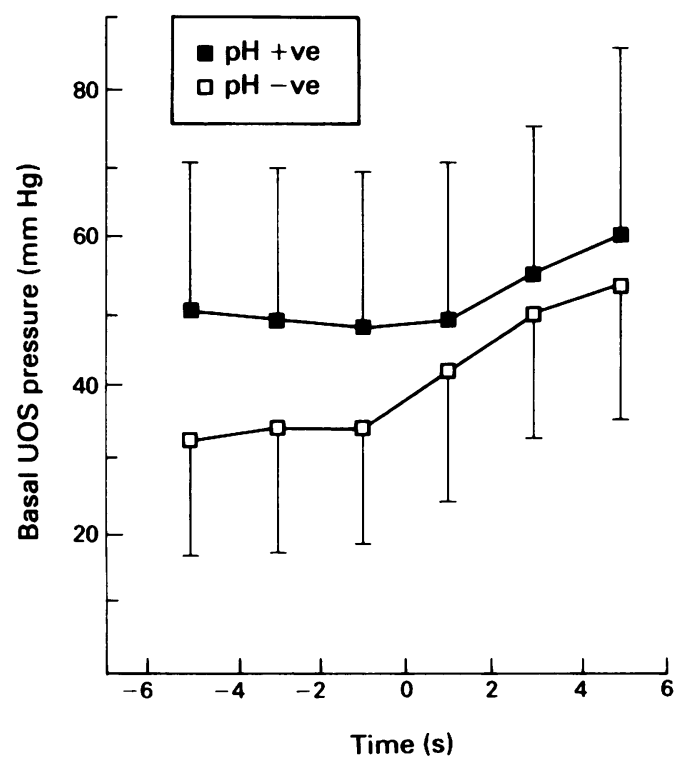

Figure 4: Measurement of UOS pressure in two second intervals from six seconds before the onset of the common cavity episode to six seconds after the onset. Episodes associated with $p H$ change are shown with filled squares, those with no $p H$ change are shown with open squares. Values are means $(S D)$ deviations are shown. 

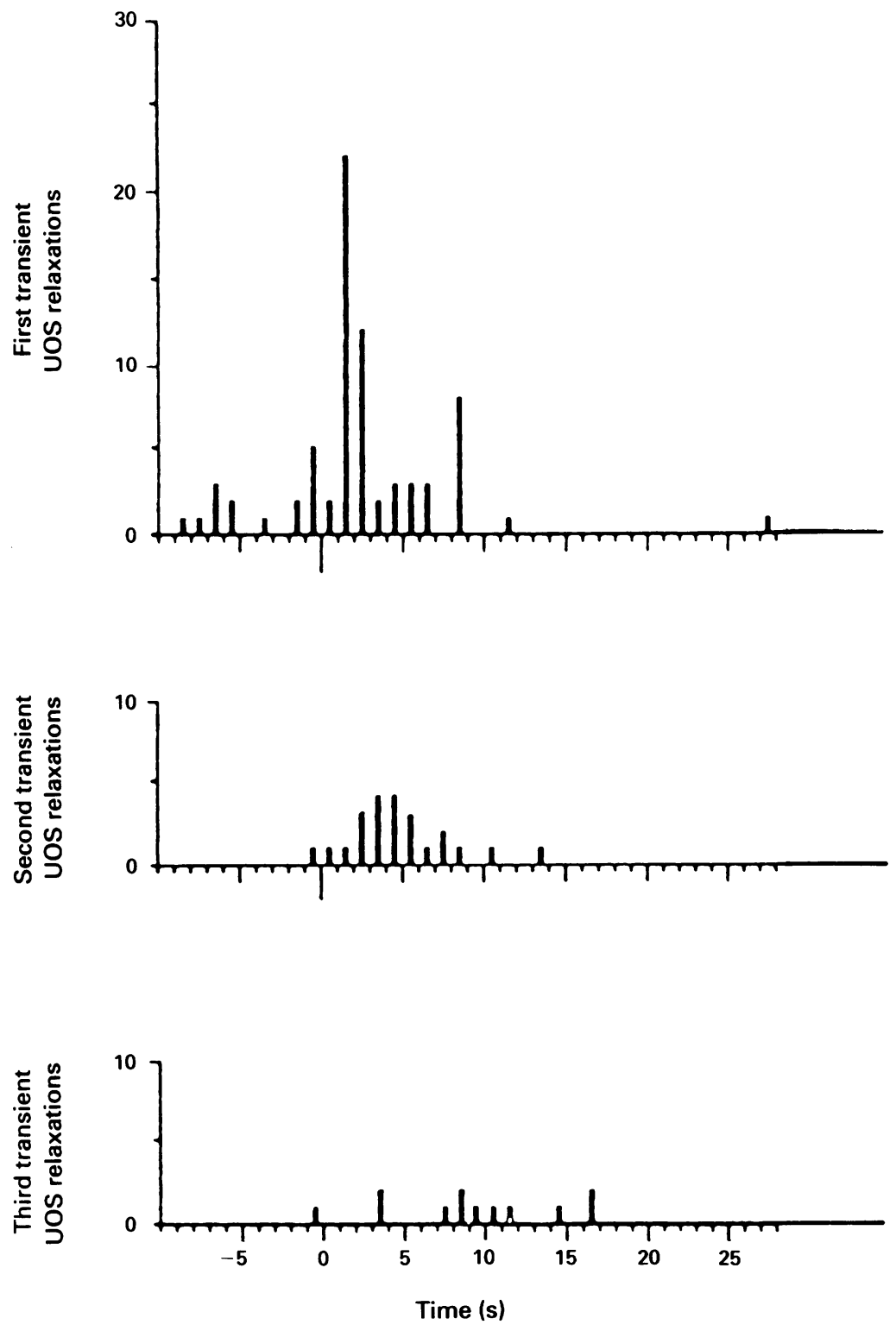

Figure 5: Timing of transient UOS relaxations with respect to the onset of the common cavity episodes. vals after the common cavity onset. The median duration of the common cavities was eight seconds (interquartile range four to 10 seconds). The median rise in basal intraoesophageal pressure by common cavity episodes was six $\mathrm{mm} \mathrm{Hg}$ (interquartile range $5-8 \mathrm{~mm} \mathrm{Hg}$ ).

Figure 4 shows the pattern of basal UOS

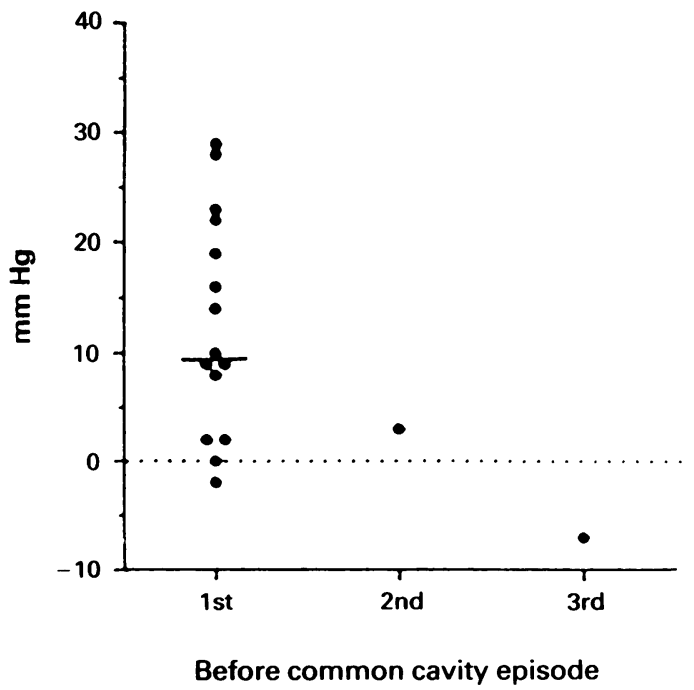

pressure before and during the common cavity episode.

Overall, common cavity episodes caused a $25 \%$ increase in mean UOS pressure from 36.5 (SD $18 \mathrm{~mm} \mathrm{Hg}$ to 48.5 (18) $\mathrm{mm} \mathrm{Hg}$, which was highly significant $(\mathrm{p}<0 \cdot 0001)$. This effect did not depend on oesophageal acidification as it occurred to the same extent with both $\mathrm{pH}^{+}$and $\mathrm{pH}^{-}$common cavity episodes. As only three episodes of acid reflux were seen in the absence of a common cavity episode no statement could be made about the effects of acidification alone.

\section{TRANSIENT UOS RELAXATIONS}

One hundred and one transient UOS relaxations occurred around the time of $60(54 \%)$ of the 112 common cavity episodes. Figure 5 shows their distribution with time; $49 \%$ occurred in the first four seconds after the onset of the common cavity episode and $34 \%$ were scattered over the 5 th to the 27th second after the onset of the common cavity. The remaining $17 \%$ occurred over the 10 seconds preceding the onset of the common cavity episode, being evenly distributed through this time. Most common cavity episodes had a single transient UOS relaxation, but up to five were seen during a single episode. There were 65 first transient UOS relaxations, 24 second transient UOS relaxations, and 12 third and subsequent transient UOS relaxations.

No grouping of the patients according to symptoms or the presence or absence of neurological deficit showed any difference in incidence of transient UOS relaxations.

Figure 6 shows the range of individual nadir pressures of transient UOS relaxations before and after the common cavity episode. The nadir pressures of the first transient UOS relaxations that occurred during the common cavity episodes differed significantly from the first transient UOS relaxations that occurred before the common cavity $(p<0.05)$. The duration of the nadir could only be graded into coarse divisions of less than one second, one to two seconds, or more than two seconds as the paper speed at which the recordings were made did not allow greater accuracy. Eighty two per cent lasted less than one second, $16 \%$ were one to two seconds, and $2 \%$ were more than two seconds in

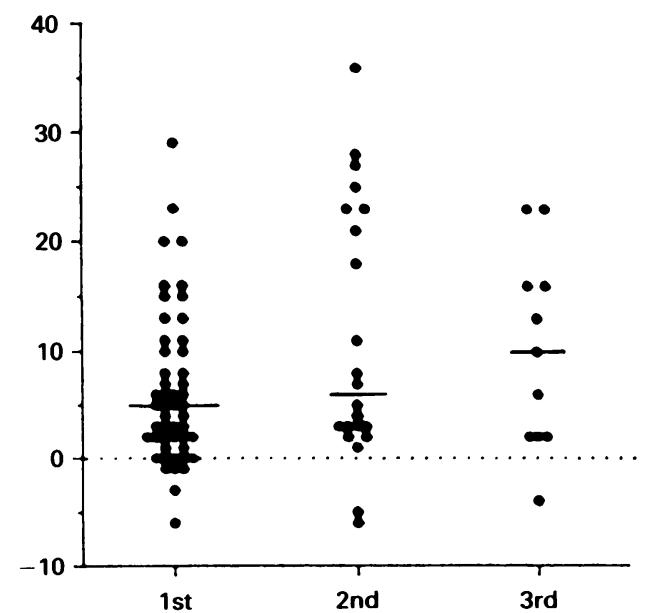

After common cavity episode 
TABLE IV Relation between increasing oesophageal body pressure and triggering of transient UOS relaxations

$\begin{array}{lllll}\text { Oesophageal body pressure }(\mathrm{mm} \mathrm{Hg}) & 3-5 & 6-8 & 9-11 & 12-21\end{array}$ \begin{tabular}{lllll}
19 & 33 & 11 & 2 \\
\hline
\end{tabular}$\quad \begin{array}{lll}18 & 20 & 8\end{array}$

There was no significant difference between the numbers that triggered transient UOS relaxations and those that did not.

TABLE V Relation between percentage of time oesophageal body pressure and gastric pressure are identical, and transient UOS relaxations

\begin{tabular}{lllr}
\hline Length of time with communication (\%) & $0-49$ & $50-99$ & 100 \\
No of episodes with relaxations & 19 & 33 & 11 \\
No of episodes without relaxations & 18 & 20 & 8 \\
\hline
\end{tabular}

There was no significant difference between the numbers that triggered transient UOS relaxations and those that did not.

duration. There were no significant differences in the mean duration of the nadirs of transient UOS relaxations according to their timing relative to the common cavity onset among the symptom groups.

Oesophageal acidification had no effect on triggering of transient UOS relaxations as the proportions of acid negative (48 of 84 ) and acid positive (14 of 22) episodes that triggered transient UOS relaxations did not differ significantly.

Neither of the measures of the extent of oesophageal distention showed any correlation with triggering of transient UOS relaxations. Table IV shows the range of increases in intraoesophageal body pressure from the common cavity episodes. Table $\mathrm{V}$ shows the percentage of time the oesophageal and gastric tracings indicated identical pressure recordings. In all but one of the common cavity episodes the pressure increase was seen in all oesophageal body monometric recording ports.

\section{Discussion}

This report describes for the first time the changes of UOS pressure that occur during spontaneous gastro-oesophageal reflux in unsedated children. One major finding in the children studied, who had a range of clinical problems, was that transient UOS relaxations occurred during a proportion of episodes of gastro-oesophageal reflux induced distention of the oesophagus. Another significant finding was that there was no difference in basal UOS pressure in children whose problems could be ascribed to oesophagopharyngeal reflux when compared with children in other groups. These findings suggest that lowered basal UOS tone is unlikely to be a major cause of oesophagopharyngeal reflux, but that transient relaxations of the UOS in response to distention are a more probable cause of such reflux. Our analyses also support further our previous finding that basal UOS pressure is highly labile and emphasise that the level of arousal must be recorded and controlled for any valid comparisons of basal UOS pressure.

The scope of the evaluations undertaken was only possible through the use of perfused sleeve manometry of the UOS. The modifications of this approach for use in children have been presented previously. ${ }^{6}$ Earlier manometric studies of the UOS in children ${ }^{23}$ used perfused sidehole pull through methods with sedation, an approach that only gives a few sample values of UOS pressure and these are influenced by the effects of sedation and stress. Our technique permits continuous recording from the upper oesophageal sphincter for periods of several hours without resort to sedation. This approach enabled us to evaluate UOS function under standard conditions with adequate control for the influence of level of arousal, and also provided the opportunity to analyse events around the time of occurrence of spontaneous episodes of gastro-oesophageal reflux.

There is a prevailing theory that basal hypotonia of the UOS is the cause of pathological oesophagopharyngeal reflux. The previous paediatric studies of Sondheimer ${ }^{2}$ and Staiano et $a l^{3}$ did not support this theory. The sleeve sensor recordings of basal UOS pressure in the present study produced many hours of UOS pressure recording and allowed us to undertake a searching and carefully controlled evaluation of the possibility that UOS hypotonia is related to any particular symptom pattern in children. The patients were classified in two ways: firstly into four groups based on their most prominent presenting symptom, and secondly according to the presence or absence of neurological deficit. The group of children with symptoms of vomiting (27 of 29) or failure to thrive (two of 29) were the group whose problems were primarily due to regurgitation. When the level of arousal was taken into account there was no significant difference of UOS pressure in this group compared with any of the other three symptom groups. The existence of a neurological deficit was also shown to have no significant effect on basal UOS pressure. We therefore support the findings of Sondheimer ${ }^{2}$ and Staiano et $a l^{3}$ that basal hypotonia of the UOS is not a cause of pathological oesophagopharyngeal reflux. Unfortunately, in the present study it was only possible to make comparisons among symptomatic children, because ethical constraints precluded measurement of UOS pressure from children who were well.

Because of the perceived significance of augmentation of basal UOS pressure as a mechanism that may prevent gastro-oesophageal reflux, we performed an analysis of the effects of oesophageal distention and acidification on basal UOS pressure. Although there is general agreement that distention of the oesophagus causes an increase in basal UOS pressure, ${ }^{511}$ there is confusion as to the effects of oesophageal acidification on basal UOS pressure. ${ }^{21112}$ In the present study distention of the oesophagus alone caused a significant increase of $12 \mathrm{~mm} \mathrm{Hg}$, and concurrent acidification had no added effect. Although the post-reflux augmentation of UOS pressure is statistically highly significant, in absolute terms it is a modest effect and we doubt that it is of great importance for the prevention of oesophagopharyngeal reflux. There were only three episodes of acid gastro-oesophageal reflux without associated oesophageal distention, too few to allow us to comment on the effects of acidification alone.

Our studies were designed to determine 
whether transient UOS relaxations occur during gastro-oesophageal reflux. Such relaxations are seen in healthy adults after abrupt oesophageal distention with insufflated gas or gas gastrooesophageal reflux. They are the basis for the audible component of belching through oesophagopharyngeal passage of gas $^{5}$ and occur independently of swallowing, having a somewhat longer time course than the UOS relaxation induced by swallowing. Our studies provided evidence of such relaxations during episodes of spontaneous gastro-oesophageal reflux in children. Transient UOS relaxations were clustered predominantly into the first few seconds of oesophageal distention consistent with the triggering of transient UOS relaxations reported by Kahrilas et al in adults. ${ }^{5}$ Acidification of the oesophagus in the presence of distention had no effect on triggering of transient UOS relaxations as seen by the proportions of common cavity episodes with and without acidification that were associated with transient UOS relaxations. This confirms a recent report by Vakil et $a l^{13}$ that there was no correlation between oesophageal acidification and occurrence of transient UOS relaxations in normal volunteers or in patients with oesophagitis. There were no transient UOS relaxations in the three episodes of acidification of the oesophagus without associated common cavity episodes, but this sample is too small to allow for any firm conclusions.

The analysis of the magnitude of the increase of basal intraoesophageal pressure produced by the common cavity and the percentage of time that the stomach was in pressure communication with the oesophageal lumen were attempts to determine whether intensity of distention had any bearing on the triggering of transient UOS relaxations. Neither of these relatively crude measures suggest that there was any correlation of degree or duration of oesophageal distention with triggering of transient UOS relaxations. Our results should not be interpreted as excluding this possibility as Kahrilas $e t a l^{5}$ did find such a dose response relation in adults, but also considerable variation in thresholds for triggering of transient UOS relaxations among different adult subjects. There is a possibility that transient UOS relaxations may be unusually easy to trigger in some subjects and so predispose them to oesophagopharyngeal reflux. This theory requires direct examination by correla- tion of events after spontaneous episodes of gastro-oesophageal reflux in which the volume of refluxate is monitored scintigraphically, or by standardised testing of thresholds for transient UOS relaxations in infants by air insufflation.

Our study has monitored UOS pressure in unsedated children and established the occurrence of transient UOS relaxations in children, which may contribute to oesophagopharyngeal reflux. Basal UOS pressure is very labile, responding to the child's level of arousal and to oesophageal distention, but not to oesophageal acidification. The prevailing theory that oesophagopharyngeal reflux is due to lowered basal UOS tone has not been supported. Definitive evaluation of the possible causes of oesophagopharyngeal reflux awaits the recording of UOS pressure during well defined episodes of such reflux. A smaller catheter to enable us to study infants in the first two months of life is eagerly awaited, as this is the age group in which most oesophagopharyngeal reflux occurs.

This work was supported by a project grant from the National Health and Medical Research Council of Australia. We thank Drs W D A Ford, T Pouras, and D J Moore for referring their patients to us.

1 Gerhardt DC, Castell DO, Winship DH, Shuck TJ Esophageal dysfunction in esophageal regurgitation. Gastroenterology 1980; 78: 893-7.

2 Sondheimer JM. Upper esophageal sphincter and pharyngoesophageal motor function in infants with and without esophageal motor function in infants with and without

3 Staiano A, Cucciara S, De Visia B, Andreotti MR, Auriccio S. Disorders of upper esophageal sphincter motility in children. F Pediatr Gastroenterol Nutr 1987; 6: 892-8.

4 Kahrilas PJ, Dent J, Dodds WJ, Hogan WJ, Arndorfer RC. A method for continuous monitoring of upper esophagea sphincter pressure. Dig Dis Sci 1987; 32: 121-8.

5 Kahrilas PJ, Dodds WJ, Dent J, Wyman JB, Hogan WJ, Arndorfer RC. Upper esophageal sphincter function during belching. Gastroenterology 1986; 91: 133-40.

6 Davidson GP, Dent J, Willing JK. Monitoring of upper oesophageal sphincter pressure. Gut 1991; 32: 607-11.

7 Frankenburg WK, Dodds JB. The Denver Developmental Screening Test. F Pediatr 1967; 71: 181 .

8 Dent J, Dodds WJ, Freidman RH, et al. Mechanism of gastrooesophageal reflux in recumbent asymptomatic human oesophageal reflux in recumbent asy
subjects. F Clin Invest 1980; 65: 256-67.

9 McNally EF, Kelly JE, Ingelfinger FJ. Mechanism of belching: effects of gastric distention with air. Gastroenterology 1964; 46: 254-9.

10 Weiner BJ. Statistical principles in experimental design. 2nd ed. Kogakusha: McGraw-Hill, 1962, 210-18 and 445-9.

11 Gerhardt DC, Shuck TJ, Bordeaux RA, Winship DH. Human upper esophageal sphincter pressure, response to volume, osmotic and acid stimuli. Gastroenterology 1978; 75: 268-74.

12 Kahrilas PJ, Dodds WJ, Dent J, Haeberle B, Hogan WJ, Arndorfer RC. Effect of sleep, spontaneous gastrooesophageal reflux and a meal on upper esophageal sphincter pressure in $466-71$.

13 Vakil N, Kahrilas PJ, Dodds W, Vanagunas A. Absence of an upper esophageal sphincter response to acid reflux. $A m \mathcal{F}$ Gastroenterol 1989; 84: 606-10. 